\title{
ASPECTOS DE QUALIDADE FÍSICO-QUÍMICA DE DOCE DE LEITE DE BÚFALAS DA RAÇA MURRAH, A PARTIR DE LEITE FRESCO E ARMAZENADO
}

\author{
Vanessa Raikelly Jacob; Claudiane Martins Roque ${ }^{2}$; Alanna do Socorro Lima da Silva ${ }^{3}$; Kedson Alessandrini Lobo \\ $\mathrm{Neve}^{4}$; Fabrizia Sayuri Otani ${ }^{5}$.
}

\footnotetext{
'Universidade Federal do Oeste do Pará, Santarém, Pará, Brasil, vanessa_star@live.com.pt 2Universidade Federal do Oeste do Pará, Santarém, Pará, Brasil, claudiane_lovely@hotmail.com 3Universidade Federal do Oeste do Pará, Santarém, Pará, Brasil, aslsilva@yahoo.com.br ^universidade Federal do Oeste do Pará, Santarém, Pará, Brasil, kedson_neves@hotmail.com 5Universidade Federal do Oeste do Pará, Santarém, Pará, Brasil, fabrizia_otani@yahoo.com.br
}

RESUMO: O presente trabalho objetivou avaliar alguns aspectos de qualidade físico-química do doce de leite de búfala pastoso, comparando como matéria-prima leite fresco e leite armazenado por nove meses. Para o desenvolvimento do trabalho, o leite após ordenha foi transportado para a Universidade Federal do Oeste do Pará - UFOPA, coado e pasteurizado. Parte do leite fresco foi utilizada, para produção imediata do doce de leite, e parte foi congelado em freezer a $-18^{\circ} \mathrm{C}$, e posteriormente processado. Para determinação da qualidade físico-química, foram analisados $\mathrm{pH}$, umidade, cinzas e acidez em ácido lático, comparando dois tratamentos (tratamento 1: doce de leite a partir de leite fresco e tratamento 2: doce de leite a partir leite armazenado), com 6 repetições cada, para todas as análises. Os dados foram submetidos à comparação de médias, aplicando-se o teste $T(P<0.05)$. Obteve-se valores de 7,02 e 6,87 de pH; 3,54 e 5,21\% umidade; 1,46 e 1,76\% para cinzas e 3,61 e 3,24 \% de ácido lático, para acidez, nos tratamentos 1e 2, respectivamente. Os doces de leite de búfala correspondentes aos tratamentos 1e 2, apresentam características físico-química dentro dos padrões vigentes preconizados pela legislação brasileira. $O$ presente estudo apresentou resultados em que é viável a utilização de leite fresco ou armazenado de qualidade para a elaboração de doce de leite de búfalas, em relação a aspectos de qualidade físico-química. Além disto, os resultados obtidos com o presente estudo podem vir a servir como parâmetros para futuros trabalhos com doce de leite de búfala.

PALAVRAS-CHAVE: Bubalinocultura, Tecnologia de alimentos, Vida de prateleira. 


\title{
PHYSICOCHEMICAL QUALITY ASPECTS OF MURRAH BUFFALO MILK CARAMEL SPREAD, FROM FRESH AND STORED MILK
}

\begin{abstract}
The aim of this work was to evaluate some physicochemical quality aspects of Murrah buffalo milk caramel spread, from fresh and stored milk for nine months. For the work, the milk after milking was transported to the Federal University of the West of Pará - UFOPA, pasteurized and pasteurized. Part of the fresh milk was used to immediately produce the buffalo milk caramel spread, and part was frozen in a freezer at $-18^{\circ} \mathrm{C}$, and then processed. To determine the physicochemical quality, $\mathrm{pH}$, moisture, ash and acidity in lactic acid were analyzed by comparing two treatments (treatment 1: fresh milk and 2: stored milk), with 6 replicates each, for all analyzes. The data were submitted to the comparison of means, applying the test $\mathrm{T}(\mathrm{P}<0.05)$. Values of $\mathrm{pH} 7.02$ and $\mathrm{pH} 6.87$ were obtained; 3.54 and $5.21 \%$ humidity; 1.46 and $1.76 \%$ for ash and 3.61 and $3.24 \%$ of lactic acid for acidity in treatments 1 and 2 , respectively. The buffalo milk caramel spread corresponding to the treatments 1 and 2, present physicochemical characteristics within the current standards recommended by the Brazilian legislation. The present study presented results in which it is feasible to use fresh or stored quality milk for the elaboration of buffalo milk caramel spread in relation to aspects of physicochemical quality. In addition, the results obtained with the present study may serve as parameters for future work with buffalo milk caramel spread.
\end{abstract}

KEYWORDS: Buffalo farming, Food technology, Shelf life.

\section{ASPECTOS DE CALIDAD FÍSICO-QUÍMICA DE DULCE DE LECHE DE BÚFALAS MURRAH, A PARTIR DE LECHE FRESCA Y ALMACENADA}

RESUMEN: El presente trabajo objetivó evaluar algunos aspectos de calidad físico-química del dulce de leche de búfala pastosa, comparando como materia prima leche fresca y leche almacenado por nueve meses. Para el desarrollo del trabajo, la leche después de ordeño fue transportada a la Universidad Federal del Oeste de Pará - UFOPA, cocido y pasteurizado. La parte de la leche fresca se utilizó para la producción inmediatamente del dulce de leche, y parte se congeló en congelador a $-18^{\circ} \mathrm{C}$ y posteriormente procesado. Para la determinación de la calidad físico-química, se analizaron pH, humedad, cenizas y acidez en ácido láctico, comparando dos tratamientos (tratamiento 1: dulce de leche con leche fresca y tratamiento 2: dulce de leche con leche almacenada), con 6 repeticiones cada una para todos los análisis. Los datos fueron sometidos a la comparación de promedios, aplicándose la prueba $T(P<0.05)$. Se obtuvieron valores de 7,02 y 6,87 de pH; 3,54 y 5,21\% de humedad; 1,46 y 1,76\% para cenizas y 3,61 y 3,24\% de ácido láctico, para la acidez, en los tratamientos 1 y 2, respectivamente. Los dulces de leche de búfala correspondientes a los tratamientos 1e 2, presentan características 
físico-químicas dentro de los estándares vigentes preconizados por la legislación brasileña. El presente estudio presentó resultados en que es viable la utilización de leche fresca o almacenada de calidad para la elaboración de dulce de leche de búfalas en relación a aspectos de calidad físico-química. Además, los resultados obtenidos con el presente estudio pueden servir como parámetros para futuros trabajos con dulce de leche de búfala.

PALABRAS CLAVE: Bubalinocultura, Tecnología de alimentos, Vida de estante.

A bubalinocultura brasileira apresentou, em 2009, um efetivo de aproximadamente 3.000.000 de cabeças, o que torna a criação de búfalos no Brasil como a mais expressiva localizada fora do continente asiático (ANUALPEC, 2009).

Dentre as raças bubalinas que foram introduzidas no Brasil, as que foram reconhecidas oficialmente pela Associação Brasileira de Criadores de Búfalos são as raças: Murrah, Jafarabadi, Mediterrâneo e Carabao, porém podem ser encontrados em todo território nacional muitos animais sem raça definida (SRD), contudo podemos encontrar exemplares de raça pura como a Murrah, Mediterrâneo, Jafarabadi e Carabao em toda a extensão territorial (ROSA et al., 2007).

Nos últimos cinquenta anos, houve um aumento da produção de leite de búfala que foi de 301,0\%, ao contrário do leite de vaca que nesse mesmo período obteve apenas 59,3\%, o de leite de cabra 85,0\% e o de ovelha 54,5\%, o que mostra incontestavelmente a importância da evolução da bubalinocultura leiteira (JORGE et al., 2011). Com o crescimento da bubalinocultura leiteira no Brasil, a produção de leite de búfala e seus derivados vêm ganhando importância, mesmo sem ainda existir uma legislação federal para regulamentar a padronização das características e da qualidade do leite e de seus derivados (GREGORY et al., 2014).

O leite desses animais apresenta elevado valor nutricional, alto teor de gordura, proteína e minerais, portanto, com aproveitamento industrial efetivamente extraordinário. Pode ser utilizado tanto para consumo in natura ou como matéria prima para elaboração de diversos derivados, tais como, iogurte, doce de leite e queijo. 
Devido à sua alta atividade de água e substrato excelente para o desenvolvimento microbiológico, a qualidade do leite tem grande importância para a indústria de laticínios visando à saúde e bem-estar dos consumidores (FIGUEIREDO et al., 2011).

No Brasil, a produção de doce de leite é bem variada, que pode variar desde fabricações artesanais a produções industriais em larga escala; e um produto encontrado em todo o país. Essa diversidade implica, muitas vezes, falta de uniformidade e padronização. Percebe-se uma grande importância dos produtos lácteos, em especial do doce de leite, para o mercado nacional. No ano de 2012, a exportação desse produto atingiu cerca de 143 toneladas, ao valor de 445,6 milhões de dólares, enquanto a importação somou, aproximadamente, seis toneladas e um milhão de dólares (MILKPOINT, 2013).

De acordo com BRASIL (1997), entendese por doce de Leite, o produto resultante da cocção de leite com açúcar, podendo ser adicionado de outras substâncias alimentícias permitidas, até concentração conveniente e parcial caramelização. Sobre as características organolépticas o doce de leite tem aspecto próprio, cor amarelada ou amarelo-pardacenta, cheiro próprio e sabor doce.

O doce de leite deve ser fabricado com matéria-prima sã e limpa, isentas de materiais terrosos, parasitos e em perfeito estado de conservação. O leite empregado deve apresentar-se normal e fresco. No preparo do produto, o leite deve entrar na proporção mínima de três partes de leite para uma de açúcar, e não pode conter substâncias estranhas à sua composição normal, além das previstas em norma (BRASIL, 1977).

O tratamento térmico aplicado na fabricação do doce de leite está sujeito a reações de escurecimento, as quais ocorrem não apenas durante 0 processamento, mas também ao longo do armazenamento, sendo um importante fator de determinação de sua qualidade (CHEN et al., 2009).

Dentre as reações de escurecimento existentes, tem destaque a reação de Maillard, a qual tende a ocorrer em uma 
grande variedade de alimentos, como no doce de leite, causando importantes alterações de cor, sabor, valor nutricional, propriedades antioxidantes e textura do alimento. São fatores determinantes para o curso da reação o processamento a temperaturas acima de $40^{\circ} \mathrm{C}$, o pH entre 6 e 8 e a atividade de água de 0,4 a 0,7 (SHIBAO; BASTOS, 2011).

O conhecimento das propriedades físico-químicas e reológicas dos alimentos é necessário, uma vez que essas propriedades são úteis no controle de qualidade dos produtos, definições de transporte e condições de armazenamento. A determinação das características de textura dos alimentos em geral é de extrema importância, uma vez que essa propriedade tem a capacidade de afetar a percepção e aceitação dos consumidores (REZAIE et al, 2011).

O presente trabalho foi desenvolvido com o objetivo de comparar alguns aspectos físico-químicos de qualidade de doce de leite de búfala pastoso, feito a partir de leite fresco e leite armazenado, como forma de verificar a viabilidade da utilização de ambos os leites para a elaboração de doce de leite, e assim otimizar o uso da matéria-prima.

O presente trabalho foi realizado no Laboratório de Tecnologia de Produtos de Origem Animal - LTPOA e de Bromatologia, do Instituto de Biodiversidade e Florestas - IBEF, da Universidade Federal do Oeste do Pará UFOPA.

Para elaboração do doce de leite, foi utilizado leite de búfalas da raça Murrah, obtido na região oeste do Pará. Logo após a ordenha, o leite foi transportado para o laboratório de Tecnologia de Produtos de Origem Animal - LTPOA, e processado, em que foi coado para a retirada de contaminantes físicos, e posteriormente pasteurizado, por meio de pasteurização lenta. Após a pasteurização parte do leite foi utilizada para elaboração do doce de leite e outra parte foi armazenada e congelada em freezer a $-18^{\circ} \mathrm{C}$, por nove meses, e posteriormente utilizado para produção de doce de leite. $O$ doce de leite foi feito utilizando leite e açúcar refinado comercial, com a proporção de 5:1,5, sendo 
cinco litros de leite para um $1,5 \mathrm{~kg}$ de açúcar, em processo de cozimento por oito horas, em fogão doméstico. Todas as análises foram feitas em seis repetições.

Foram feitas análises de umidade e cinzas, por meio de secagem em estufa a $105^{\circ} \mathrm{C}$ e carbonização em mufla a $550^{\circ} \mathrm{C}$, respectivamente, segundo metodologias propostas pelo Instituto Adolfo Lutz - IAL (2008), para leite e derivados. O pH do doce de leite foi determinado utilizando-se o método eletroanalítico usando o peagâmetro eletrônico, com termômetro para aferir a temperatura, em conformidade com o Instituto Adolfo Lutz - IAL (2008). E para determinar acidez em ácido lático, foi utilizada metodologia descrita pelo Instituto Adolfo Lutz - IAL (2008).

Os dados obtidos foram tabulados em planilha do programa Microsoft Excel ${ }^{\circledR}$, do pacote de programas Microsoft Office ${ }^{\oplus}$. Os dados foram submetidos para comparação por meio do programa estatístico ASSISTAT $^{\oplus}$ versão 7.7 , em que foram verificadas as pressuposições de análise e comparação de médias pelo teste de t, em $5 \%$ de probabilidade.
Os resultados das análises de $\mathrm{pH}$, umidade, cinzas e acidez do doce de leite de búfala com leite fresco e armazenado encontram-se descritos na Tabela 1.

A avalição das características físicoquímica de um alimento é importante para realizar a caracterização e classificação do mesmo. No presente trabalho, as interpretações dos resultados foram feitas com parâmetros de doce de leite bovino, pois ainda não há uma legislação específica para o doce de leite bubalino.

A análise de comparação de médias entre as amostras, mostrou haver diferença significativa $(p<0,05)$ nos teores de $\mathrm{pH}$, umidade e cinzas entre os tratamentos (Tabela 1). Porém observou-se que a acidez não apresentou diferença estatística entre os tratamentos 1 e 2 .

O Ministério da Agricultura, Pecuária e Abastecimento (MAPA) estabelece valores físico-químicos máximos que devem ser obrigatoriamente seguidos durante a formulação do doce de leite. A uniformidade e homogeneidade dificilmente são obtidas durante a fabricação do doce de leite, este produto 
apresenta variação principalmente em

(DEMIATE et al., 2001 citado por VIEIRA et relação às características físico-químicas, al., 2011). composicionais, sensoriais e nutricionais

Tabela 1. Resultados de médias \pm desvio padrão de pH, umidade, cinzas e acidez do doce de leite de búfala.

\begin{tabular}{ccccc}
\hline Tratamento & $\mathrm{pH}$ & Umidade (\%) & Cinzas (\%) & Acidez (\%) \\
\hline 1 & $7,02 \pm 0,04 \mathrm{a}$ & $3,54 \pm 1,24 \mathrm{a}$ & $1,46 \pm 0,01 \mathrm{a}$ & $3,61 \pm 084, \mathrm{a}$ \\
2 & $6,87 \pm 0,01 \mathrm{~b}$ & $5,21 \pm 0,82 \mathrm{~b}$ & $1,76 \pm 0,09 \mathrm{~b}$ & $3,24 \pm 0,003 \mathrm{a}$ \\
\hline
\end{tabular}

Tratamento 1= doce de leite de búfala a partir leite fresco; tratamento 2 = doce de leite de búfala a partir leite armazenado do por nove meses. Médias seguidas de letras iguais nas colunas não diferem estatisticamente entre si pelo teste $t(P<0.05)$.

O pH apresentou variações nos valores, sendo que no tratamento 1 apresentou diferença $(p<0,05)$ para este parâmetro quando comparados com o tratamento 2. Em estudo realizado por Milagres et al. (2010) em análise físico-química e sensorial de doce de leite produzido sem adição de sacarose, foram encontrados valores de $\mathrm{pH}$ em torno de 6,39\%, sendo semelhantes aos valores encontrados neste estudo (Tabela 1). Porém não há em legislação um parâmetro específico de pH ideal para doce de leite.

Os resultados obtidos no presente trabalho mostram que, para o teor de umidade apresentaram diferença significativa $(p<0,05)$, porém ambos encontram-se dentro dos padrões, dos valores determinado pela Portaria $n^{\circ}$ 354/1997 do MAPA, que preconiza um máximo de 30\% de umidade para doce de leite.

Os teores de cinzas, de acordo com a legislação do MAPA, devem apresentar valores máximos de 2\%. No presente trabalho, os resultados obtidos estão dentro do limite exigido. O que contrapõe com os resultados obtidos por Guimarães et al. (2012) em análise de amostra de doce de leite feito em seu trabalho exibiu valores 
acima do proposto pelo MAPA, com valores entre $2,15 \%$ e 2,84\%. Já nos resultados obtidos por Oliveira et al. (2010), foi revelado que todas as amostras estudadas apresentaram valores entre 1,34 a 1,77\%, o que esta em conformidade com o estabelecido por lei vindo ao encontro dos valores obtidos no presente trabalho.

A acidez em ácido lático do doce de leite de búfala nos tratamentos 1 e 2 , apresentou os valores de 3,2 e 3,6\%, respectivamente, e ambos estão de acordo com o estabelecido pelo MAPA, que exige no máximo 5\%. Em trabalhos realizados por Oliveira et al. (2010), foram encontrados em doce de leite tradicional valores de acidez na ordem de 0,24 á 0,39\%, valores estes inferiores aos encontrados no presente estudo.

Os resultados das análises de média de umidade, cinza e acidez de doce de leite de búfala com leite fresco e armazenado, comparado com os padrões estabelecidos pelo Ministério da Agricultura encontramse descritos na Tabela 2.

De acordo com os padrões instituídos pelo Ministério da Agricultura, por meio da Portaria no 354, de 04/09/1997, o doce de leite elaborado com leite de búfala, tanto fresco assim como armazenado, encontrou- se de acordo com as características físico-químicas preconizadas pela legislação (Tabela 2).

Tabela 2. Requisitos físico-químicos obrigatórios para doce de leite de acordo com Brasil (1997).

\begin{tabular}{ll}
\hline Requisitos & Padrão (Brasil, 1997) \\
\hline Umidade (\%) & Máximo 30 \\
Cinzas (\%) & Máximo 2 \\
Acidez (\%) & Máximo 5 \\
\hline
\end{tabular}

Fonte: Adaptada do Regulamento Técnico para Fixação de Identidade e Qualidade de Doce de Leite (BRASIL, 1997).

A utilização de matéria-prima de qualidade do produto final (BRASIL, 1977). qualidade para produção de doce de leite é O presente estudo apresentou resultados exigida pela legislação, e determinante na em que é viável a utilização de leite fresco 
ou armazenado de qualidade para a elaboração de doce de leite de búfalas, em relação a aspectos de qualidade físicoquímica.

O doce de leite de búfalas, feito a partir de leite fresco ou armazenado, apresenta características físico-químicas dentro dos padrões vigentes preconizados pela legislação brasileira estabelecida para bovinos. Além disto, os resultados obtidos com o presente estudo podem servir como parâmetros para futuros trabalhos com doce de leite de búfala, usando leite fresco ou armazenado.

\section{REFERÊNCIAS}

ANUALPEC. Anuário da pecuária brasileira. FNP Consultoria/Agros Comunicação, São Paulo, SP. 2009, 400p. Disponível em: <http: //www.informaecon fnp.com/publicações/anuários/anualpec>. Acesso em: 05 abr. 2017.

BRASIL. Ministério da Agricultura, Pecuária e do Abastecimento. Portaria no 354, de 4 de Setembro de 1997. Regulamento Técnico para fixação de identidade e qualidade de doce de leite. Diário Oficial da República Federativa do Brasil, Brasília 08 set. 1997. Disponível em: < http://www.anvisa.gov.br/anvisalegis/resol/ 12_78_doce_leite.htm\# >. Acesso em: 05 jul. 2017.

CHEN, S. L., YANG, D. J., CHEN, H. Y.; LIU, S. C. Effect of hot acidic fructose solution on caramelisation intermediates including colour, hydroxymethylfurfural and antioxidative activity changes.Food chemistry, v. 114, n. 2, p. 582-588, 2009. Disponível em: <http://www.sciencedirect.com/science /article/pii/S0308814608011837>. Acesso em: 15 jun. 2017.

DEMIATE, I. M.; KONKEL, F. E.; PEDROSO, R. A. Avaliação da qualidade de amostras comerciais de doce de leite pastoso composição química. Ciência e Tecnologia de Alimentos, v. 21, n. 1, p.108-114, 2001. Disponível em: <http://www.scielo.br/pdf/\%0D/cta/v21 n1/5375.pdf>. Acesso em: 17 junh. 2017.

FIGUEIREDO, E. L.; LOURENCO JUNIOR, J. B.; TORO, M. J. U.; LIMA, S. C. G. Queijo do Marajó tipo creme: parâmetros físicoquímicos e sensoriais. Revista do Instituto de Laticínios Cândido Tostes, v. 66, n. 378, p. 26-33, 2011. Disponível em: <https://www.revistadoilct.com.br/rilct/artic le/view/152>. Acesso em: 18 set. 2017.

GREGORY, L., ROSSI, R. S., MENDES, J. P. G., NEUWIRT, N., MARQUES, E. C., MELVILLE, P. A.; MONTEIRO, B. M. Ocorrência dos principais agentes bacterianos e parasitários em fezes diarreicas de bezerros búfalos nos estados de São Paulo e Paraná. Arquivos do Instituto Biológico, v. 81, n. 2, p. 180-185, $2014 . \quad$ Disponível em: 
<http://189.126.110.61/arqib/article/view/22 493>. Acesso em: 05 julh.2017.

GUIMARÃES, Í. C. D. O., LEÃO, M. H. M. D. R., PIMENTA, C. J., FERREIRA, L. D. O; FERREIRA, E. B. Development and description of light functional dulce de leche with coffee. Ciência e Agrotecnologia, v. 36, n. 2, p. 195-203, 2012. Disponível em: <http://www.scielo.br/scielo.php?pid=S141 370542012000200008\&script=sci_arttext\&tl $\mathrm{ng}=\mathrm{pt}>$. Acesso em: 18 set. 2017.

\section{INSTITUTO ADOLFO LUTZ. Normas} Analíticas do Instituto Adolfo Lutz. Métodos químicos e físicos para análise de alimentos, v. 1, 2008. Disponível em:< http://www.sidalc.net/cgi bin/wxis.exe/?IsisScript=senasa. $x i s \&$ method $=$ post \&formato $=2 \&$ cantidad $=1 \&$ expresion $=m f n=000089>$. Acesso em: 07jun. 2017.

MILKPOINT. Balança Comercial de Lácteos de 2012. Disponível em: <Disponível em: http://www.milkpoint.com.br/cadeiado-leite/estatisticas/estatisticas. aspx> Acessado em: 01 de ago. 2013.

MILAGRES, M. P., DIAS, G., ARAÚJO MAGALHÃES, M., OTTOMAR SILVA, M.; MOTA RAMOS, A. Análise físico-química e sensorial de doce de leite produzido sem adição de sacarose. Revista Ceres, v. 57, n. 4, 2010. Disponivel em: <http://www.redalyc.org/html/3052/30522 6768001/>. Acesso em: 18 set. 2017.

OLIVEIRA, R. M. E., de Oliveira, A. R. C., Ribeiro, L. P., Pereira, R., Pinto, S. M.; de Abreu, L. R. Caracterização química de doces de leite comercializados a granel em
Lavras/MG. Revista do Instituto de Laticínios Cândido Tostes, v. 65, n. 377, p. 5-8, 2010. Disponível em:

https://www.revistadoilct.com.br/rilct/article /view/143>. Acesso em: 18 set. 2017.

PASSOS, B. Raças bubalinas. Disponível em: http://zootecniae10.blogspot.com.br/2011/1 1/racas-bubalinas.html. Acesso em: 02 abr.2017.

PREGNOLATTO, W.; PREGNOLATTO, N. P. Normas analíticas do instituto Adolfo Lutz. 2008. Disponível em: < http:www.sidalc.net/cgi bin/wxis.exe/?lsisScript=senasa.xis\&method $=$ post $\&$ formato $=2 \&$ cantidad $=1 \&$ expresion $=m f n=000089>$. Acesso em: 05 junh. 2017.

RANALLI, N.; ANDRES, S. C.; CALIFANO, A. $N$. Physicochemical and rheological characterization of "dulce de leche". Journal of texture studies, v. 43, n. 2, p. 115-123, 2012. Disponível em:< http:// onlinelibrary.wiley.com/doi/10.1111/j.17454603.2011.00321.x/full > Acesso em: 07 ago. 2017.

REZAEI, R., KHOMEIRI, M., KASHANINEJAD, M., \& AALAMI, M.Effects of guar gum and arabic gum on the physicochemical, sensory and flow behaviour characteristics of frozen yoghurt. International journal of dairy technology, v. 64, n. 4, p. 563-568, 2011. Disponível em:<http://onlinelibrary.wiley.com/doi/10.1 111/j.1471-0307.2011.00705.x/full>. Acesso em: 19 junh. 2017.

ROCHA, L. A. C. Qualidade do leite de búfala e desenvolvimento de bebidas láctea 
com diferentes níveis de iorgute e soro de queijo. 2008. Tese de Doutorado. Dissertação (Mestrado em engenharia de alimentos), Universidade Estadual do Sudoeste da Bahia, Salvador. Disponível em:<http://www2.uesb.br/ppg/ppgecal/wp content; uploads/2017/4/LUCIANAROCHA.pdf>. Acesso em: 07 ago. 2017.

ROSA, B. R. T., FERREIRA, M. M. G., AVANTE, M. L., ZANGIROLAMI FILHO, D.; MARTINS, I. Introdução de búfalos no Brasil e sua aptidão leiteira. Revista Cientifica Eletrônica de Medicina Veterinária, v. 4, n. 8, 2007. Disponível em: $<$ http:// faef.revista.inf.br/imagens_arquivos/.../vDX NCfZHc6Lxtn8_2013-5-21-17-2-38.pdf>. Acesso em: 05 agos.2017.

SHIBAO, J.; BASTOS, D. H. M. Produtos da reação de Maillard em alimentos: implicações para a saúde. Revista de Nutrição, 2011. Disponível em:<http:// www. scienceopen. com/document?id=2d7a a30e-3f17-4f86-b8ed-80428ce8c343> . Acesso em: 17 jun. 2017.

TAMIME, Adnan Y. (Ed.). Dairy powders and concentrated products. John Wiley \& Sons, 2009. Disponível em: <http:// books.google.com.br/books?hl=pt BR\&Ir=lang_es|lang_en|lang_pt\&id=TVHU2 myKicC \&oi=fnd\&pg=PR5\&dq=Dairy + pow ders+and +concentrated + products + Tamim $\mathrm{e}+\mathrm{AY}+$ \&ots=_3dszr3qBK\&sig=ksuMPHueu _04D0AFCO3ICKyesO\# $v=$ onepage\& $q=$ Dair y\%20powders\%20and\%20concentrated\%2 Oproducts\%20Tamime\%20 AY\&f=false > . Acesso em: 17 jun. 2017.
VIEIRA, M. C.; GALLINA, D. A.; CAVICHIOLO, J. R.; GOMES, R. A. R.; FACHINI, C.; ZACARCHENCO, P. B. S. Produção de doce de leite tradicional, light e diet: estudo comparativo de custos e viabilidade econômica. Informações Econômicas, v. 41, n. 10, 2011. Disponível em: <http://www.brasilsns.org.br/files/empr esas/00005010001000/641/b8f7b459710c6caba435b67c4 f619f58.pdf>. Acesso em: 17 jun.2017. 